\title{
Outcome measures to be considered on asthma in elderly
}

\author{
Sandra Nora Gonzalez-Diaz ${ }^{\mathrm{a}}$, Rosalaura Virginia Villarreal-Gonzalez ${ }^{\mathrm{a}, \mathrm{b}}$ \\ Cindy Elizabeth De Lira-Quezada ${ }^{\mathrm{a}, \mathrm{b}}$, and Rosa Ivett Guzman-Avilan ${ }^{\mathrm{a}, \mathrm{b}}$
}

\begin{abstract}
Purpose of review
Asthma is a chronic heterogeneous respiratory disease which is characterized by airflow limitation and variable respiratory symptoms. Asthma in patients more than 65 years of age has an important negative impact on quality of life. The pathophysiology and treatment of asthma in older patients are not as well identified as in younger groups of ages. In this review we intend to outline characteristics found in elderly adults which distinguish them from other age groups of patients with asthma.
\end{abstract}

\section{Recent findings}

With increasing age, there are alterations in the innate and adaptive immune responses, known as 'immunosenescence.' These age-associated modifications include an altered response after a pathogenic exposure or tissue injury, moderately mediated through an irreversible loss of cellular replication and defective tissue repair.

\section{Summary}

Asthma is a consequence of complex gene-environment interactions, with diversity in clinical presentation and the type and intensity of airway inflammation and remodeling. Age-associated changes in lung physiology and morphology may occur and contribute to asthma. Aging is correlated with a notable decrease in elastic recoil, greater chest wall rigidity, and poor respiratory muscle strength. Underreporting of symptoms by elderly patients is common because of multiple underlying causes.

Video abstract

http://links.lww.com/COAl/A17.

\section{Keywords}

asthma, elderly, immunosenescence, inflammaging, late-onset asthma, long-standing asthma, phenotypes

\section{INTRODUCTION}

Asthma is one of the most common chronic, noncommunicable diseases in children and adults, characterized by variable respiratory symptoms and airflow limitation $\left[1^{-}\right]$. Asthma is a consequence of complex gene-environment interactions, with heterogeneity in clinical presentation and the type and intensity of airway inflammation and remodeling, affecting around 334 million people [2].

As the life expectancy increases across the world, the number of people over the age of 65 years is expected to nearly triple by 2050 , reaching approximately 1.5 billion of $16 \%$ of the global population by this time [3"'].

Patients older than 55 years have higher rates of emergency department visits, hospitalization, and asthma-associated healthcare cost compared with those younger than 55. Despite this, asthma in older adults continues to be an under-recognized and undertreated epidemic [4].
Asthma in patients more than 65 years of age has a significant negative impact on measures of quality of life (QOL) [5"']. Asthma QOL has been related to environmental triggers and to individual factors such as asthma self-efficacy, asthma knowledge, asthma control, and asthma phenotype [6]. The pathophysiology and treatment of asthma in older patients are not as well characterized as they are in younger adults and children [6].

\footnotetext{
${ }^{a}$ Regional Center of Allergy and Clinical Immunology, Universidad Autonoma de Nuevo León and ${ }^{b}$ World Allergy Organization Center of Excellence; Facultad de Medicina, University Hospital 'Dr José Eleuterio González;' Monterrey, Nuevo León, Mexico

Correspondence to Sandra Nora Gonzalez-Diaz, MD, PhD, Monterrey, Nuevo León, Mexico. Tel: +52 (818) 348 2459;

e-mail: sgonzalezdiaz@yahoo.com
}

Curr Opin Allergy Clin Immunol 2019, 19:000-000

DOI:10.1097/ACl.0000000000000525 


\section{KEY POINTS}

- Prevalence of asthma in the elderly has increased in recent years.

- Always consider associated comorbidities.

- Related to elevated mortality compared with other age groups of patients with asthma.

- Characteristic alterations are present such as 'immunosenescence' and 'inflammaging.'

\section{PREVALENCE}

According to the National Health Interview Survey data, the annual prevalence of asthma in individuals who are more than 65-year old is between 4 and 13\% $\left[2,3^{-"}\right]$.

Prevalence is highest in developed countries for example, Australia (21\%) - and lowest in developing countries - for example, China $(0.2 \%)$. However, prevalence is probably substantially underestimated in resource-poor countries, where basic asthma medications are not available and patients have difficulty accessing healthcare [2,3"'].

Asthma was once considered primarily a developmental disease of childhood; however, several studies report that the lifetime prevalence is high in the elderly patient, close to $13 \%$ compared with roughly $14 \%$ in children of ages less than 18 years old and $7.5 \%$ in adults of ages $18-74$ years [ ["'].
This age group of patients has the highest rates of morbidity and mortality from its disease compared with other ages. Elderly patients with asthma are over five times more likely to die from their disease than younger individuals. Of aged patients, those who are female, Black, Hispanic, and from a lower income group are at the greatest risk for frequent hospitalizations (Fig. 1) [5"'].

\section{PATHOGENESIS}

In the elderly, changes related to aging, decreased immune function and other comorbidities common in older age are overlying on asthma. Asthma in the elderly is often associated with allergic triggers. Owing to this, the cause of asthma in the elderly is considered complex and multifactorial [7].

There are several age-associated changes in lung physiology and morphology that occur and may contribute with the presence of asthma. Aging is correlated with a notable decrease in elastic recoil, greater chest wall rigidity, and poor respiratory muscle strength [5"'].

The consequence of the age-related modifications, including poor chest wall compliance, decreased respiratory muscle strength, and the macro and microscopic changes in the lung parenchyma, are proposed to have an important role in altering pulmonary function. In particular, older patients, even without lung disease, are more propense to develop obstructive and mixed patterns with increased residual volume, and reductions in

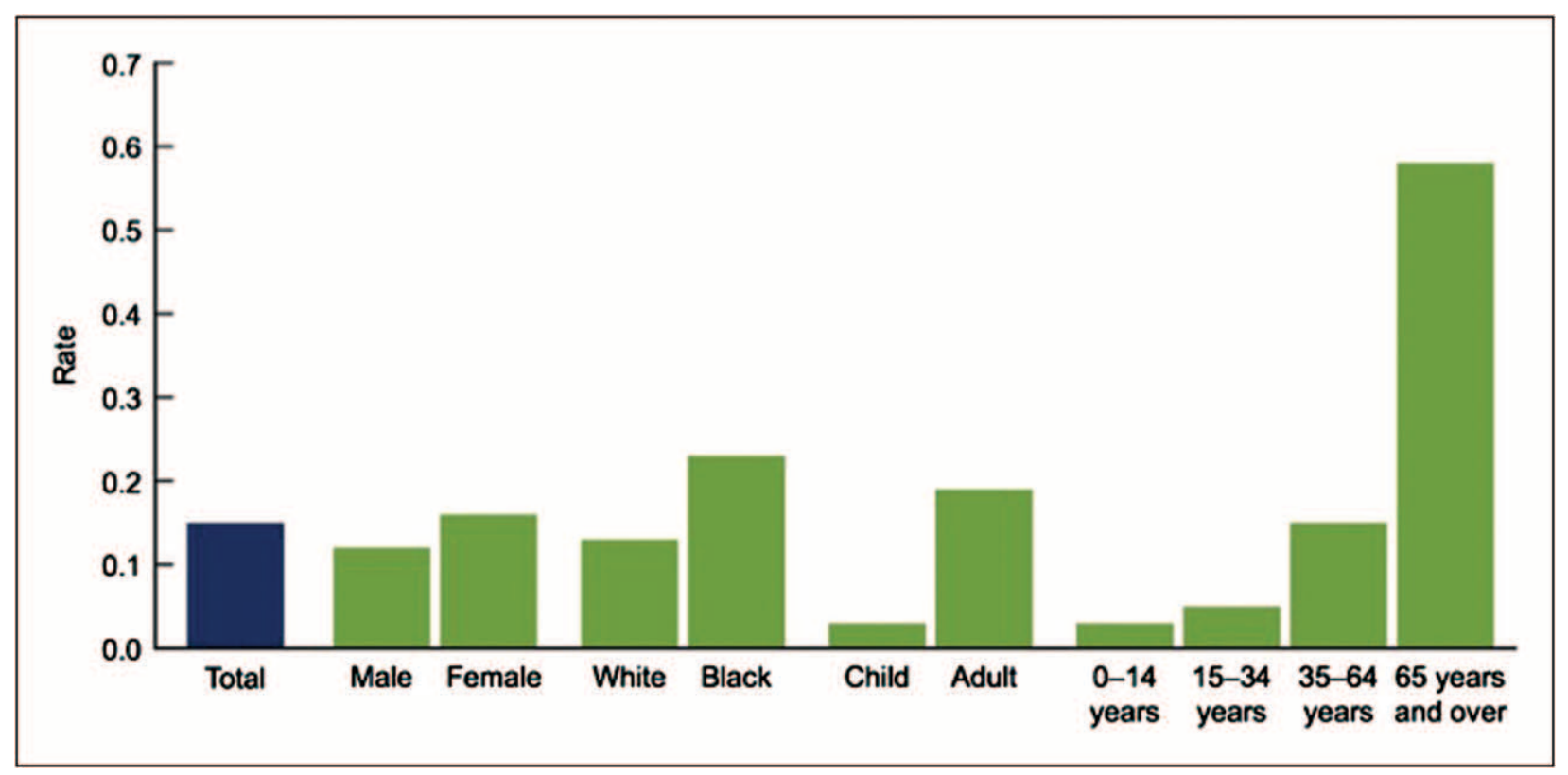

FIGURE 1. Asthma deaths per 1000 by demographic (2007-2009). Source: CDC/NCHS, National Vital Statistics System and National Health Interview Survey Data for table available at: http://www.cdc.gov/nchs/products/databriefs/db94.htm. Adapted with permission from [5"']. 
forced expiratory volume in $1 \mathrm{~s}$ (FEV1), FEV1/forced vital capacity (FVC) ratio, and FVC with a discrete change in total lung capacity [5"'].

\section{IMIMUNOSENESCENCE}

With increasing age, there are alterations in the innate and adaptive immune response, which impact the pathology and the treatment of asthma in the elderly. There are two alterations in aging in the immune responses that have been described and can occur simultaneously: The first one, 'immunosenescence,' is a 'blunted' response after a pathogenic threat or tissue injury. However, despite an inability to proliferate, some senescent cells remain alive, functioning at an altered capacity [8]. This results in the second process, 'inflammaging,' an increased low-grade basal systemic inflammation which may also result from the dysregulated activities of immune cells other than $\mathrm{CD}^{+} \mathrm{T}$ cells (e.g., IL1- $\beta$, IL- 6 , and TNF- $\alpha$ ) which tend to be pro-inflammatory as an essential part of their function [9].

The mechanisms of immunosenescence and inflammaging are the consequence of the genetic and environmental exposures, accumulation of reactive oxygen species from metabolic activity, and mutagenesis events [8].

The possible impacts of immunosenescence and inflammaging on asthma are briefly outlined in Table 1 [8]. Collectively, alterations in innate and adaptive cells likely have several clinical implications including decreasing the response to vaccinations and increasing rates of infections, which in turn may exacerbate asthma, or play a role in the inception of asthma with a later onset [8]. Furthermore, older patients with asthma may have altered airway and systemic inflammation resembling changes seen in a phenotype of severe asthma in younger patients, which is often less responsive to corticosteroid treatment [10].

\section{PHENOTYPES OF ASTHMA IN THE ELDERLY}

Elderly patients with asthma are classified upon their age of asthma onset and duration of disease into: long-standing asthma (LSA), late-onset asthma (LOA), or remitting childhood asthma that recurs at a later age. The age cutoff for the diagnosis of LOA is not persistent: more than 12 years of age in some studies and up to more than 65 years in others. LSA usually initiates before the age of 12 years. Atopy appears to have a discrete role on the evolution of LOA in comparison with LSA. Factors predicting LOA include smoking, new habitual snoring, new rhinitis symptoms, chronic sinus symptoms, weight gain, and obesity (especially among females). Although viral infections are common triggers for asthma beginning, Chlamydia pneumoniae and other atypical infections may be the cause in some patients with LOA. Patients with LOA are likely to have a higher baseline FEV1, and a marked bronchodilator response than those with LSA, who are more likely to have irreversible or partially reversible airway obstruction, and greater airway hyperinflation (Table 2) [5"'].

\section{CLINICAL PRESENTATION}

The symptoms of asthma in older adults are nonspecific, and are common to other conditions that are often seen with other comorbidities frequent in older adults [3"'].

The diagnosis of asthma in the elderly is similar to younger patients with the presence of symptoms including cough, wheezing, chest tightness, and dyspnea [7]. Usually, the symptoms are worse at night and may be triggered by physical activity. However, compared with younger asthmatics, elderly asthmatics have greater morbidity and lower scores on subjective assessments of health-related QOL. In addition to asthma symptoms, the elderly asthmatic is more likely to report poor general health, symptoms of depression, and limitation of activities of daily living [3"'].

In at least half of elderly patients, the diagnosis of asthma is recent. Factors that influence the diagnosis may be poor perception of signs and symptoms, presence of extrapulmonary manifestations, effects of aging in the respiratory tract and technical difficulties to make reliable measurements of lung function [7].

Older patients may not report their symptomatology, and may attribute breathlessness to normal aging or comorbidities such as cardiovascular disease and obesity. Underreporting of symptoms in older adults may have many causes, including fear, depression, cognitive impairment, social isolation, denial, and poor medical literacy [3"'].

Asthma is often provoked by environmental exposures. The Cardiovascular Health Study general population sample of asthmatics found that asthmalike symptoms were often brought on or made worse by exposure to the following factors: dust, smoke, and contact with animals, plants, or pollens [ $\left.3^{-"}\right]$.

A history of atopy is a strong predictor of asthma in this age group, and current or previous allergic rhinitis, sinusitis, and nasal polyps are not uncommon [3"']. The prevalence of atopic conditions has expanded in general over the past decades, which may contribute to the appearance of asthma in elderly patients. In a minor part, asthma in the 
Table 1. Impacts of immunosenescence and inflammaging on asthma

\begin{tabular}{|c|c|c|}
\hline Immune responses & Cell type & Age-associated changes \\
\hline \multirow[t]{19}{*}{ Innate } & Neutrophils & $\uparrow$ Number of cells in BALF and neutrophil elastase in those without asthma \\
\hline & & $\uparrow$ Number of cells in sputum of older vs. younger patients with asthma \\
\hline & & $\uparrow$ Number of sputum neutrophil mediators in older patients with asthma (IL-8, MMP9) \\
\hline & & $\downarrow$ Chemotaxis \\
\hline & & $\downarrow$ Secretion of neutrophil extracellular traps, which normally function to immobilize bacteria \\
\hline & & $\downarrow$ Phagocytosis \\
\hline & & $\uparrow$ Basal production of ROS and neutrophil elastase (damages local tissues) \\
\hline & & $\downarrow$ Production of ROS in response to bacterial infection \\
\hline & Eosinophils & $\uparrow$ Degranulation of peripheral cells in response to IL-5 in older patients with asthma \\
\hline & & $\begin{array}{l}\downarrow \text { Peripheral number of cells associated with development of BHR and aging in men (Normative } \\
\text { Aging Study) }\end{array}$ \\
\hline & Dendritic cells & $\uparrow I L-6$ and TNF- $\alpha$ expression and $\downarrow$ IFN- $\gamma$ \\
\hline & & $\downarrow$ Phagocytosis \\
\hline & & $\downarrow$ Toll-like receptor function \\
\hline & & $\downarrow$ Migration \\
\hline & Macrophages & $\downarrow$ Phagocytosis \\
\hline & & $\downarrow T L R$ expression \\
\hline & & $\uparrow T N F-\alpha$, IL-8 expression \\
\hline & Epithelial cells & $\downarrow$ Ciliary beat frequency \\
\hline & & $\downarrow$ Barrier function because of loss of tight cellular junctions \\
\hline \multirow[t]{10}{*}{ Adaptative } & B-lymphocytes & $\begin{array}{l}\downarrow \text { Ability to produce specific, long-lasting, and diverse antibodies to antigens (e.g., vaccination } \\
\text { and infection) }\end{array}$ \\
\hline & & $\downarrow$ Naive cells produced by the bone marrow \\
\hline & & $\downarrow$ Total $\lg E$ \\
\hline & & $\begin{array}{l}\downarrow \text { Antigen-specific lgE (however, antigen sensitization in older patients with asthma not } \\
\text { uncommon, but its effect on asthma not clear) }\end{array}$ \\
\hline & T-lymphocytes & $\downarrow$ Naive $T$ cells as thymus involutes \\
\hline & & $\uparrow$ Number of circulating $T$ cells because of increase in the number of memory $T$ cells \\
\hline & & $\downarrow$ Expression of CD154 (CD4OL), decreasing B-cell activation, and antibody class switching \\
\hline & & $\begin{array}{l}\uparrow \text { Survival of memory CD8 } p \text { cells, possibly because of chronic cytomegalovirus stimulation } \\
\text { (inverted CD4:CD8 ratio in older adults with asthma, associated with decreased asthma } \\
\text { control }\end{array}$ \\
\hline & Th17 cells & $\uparrow$ Number with aging \\
\hline & & $\uparrow$ Number in BALF of LOA animal model \\
\hline
\end{tabular}

BALF, bronchoalveolar fluid lavage; BHR, bronchial hyperresponsiveness; LOA, late-onset asthma; MMP-9, matrix metallopeptidase 9; ROS, reactive oxygen species; TLR, toll like receptor.

Adapted with permission from [8].

elderly may be associated with allergic triggers and sensitization to dust mites and cat. The patients sensitized to cockroach, may have an increased risk for asthma morbidity [7].

Results of several studies have shown that the prevalence of allergen sensitization is high, which supports that IgE-mediated mechanisms may be involved in elderly asthmatics [11].

\section{COMORBIDITIES}

Comorbidities contribute to the clinical complexity of the elderly, decrease in QOL as well as poor asthma management (Table 3) [5""]. Gemicioglu et al. [12] studied adults with asthma (age $<65$ years) and elderly patients with asthma (age $\geq 65$ years) and found that the adherence to treatment was similar for both groups, but decreased with the presence of gastritis, ulcer, gastroesophageal reflux, and coronary artery disease.

Specific comorbidities such as patients with dementia, orthodontics, and arthritis, may contribute to the adherence of treatment. Yáñez et al. [13] found that allergic comorbidities were most frequently found $(65 \%)$, followed by hypertension (45\%), gastroesophageal reflux disease (16\%), 
Table 2. Phenotypes of asthma among elderly

\begin{tabular}{lll}
\hline & Late-onset asthma & $\begin{array}{l}\text { Long-standing } \\
\text { asthma }\end{array}$ \\
\hline Age of onset & $\begin{array}{c}\text { Variable but typically } \\
\text { as an adult } \\
\text { Higher }\end{array}$ & Age $<12$ \\
FEV1 & Variable & Lower \\
Severity & Variable & Variable \\
TH2-mediated & $(-)$ & $(+)$ \\
Atopy & $(-)$ & $(+)$ \\
Family history & $(+)$ & $(+)$ \\
Obesity & $(+)$ & $(-)$ \\
Tobacco use & & $(-)$ \\
\hline
\end{tabular}

FEV1, forced expiratory volume in $1 \mathrm{~s}$.

Adapted with permission from [5"'].

diabetes (12\%), obesity (9\%), coronary diseases (8\%), osteoarthritis, arthritis (7\%), hypothyroidism (7\%), and dyslipidemia (3\%).

There are other important comorbidities such as frequent falls, which are a problem for elderly patients. Bozek et al. [14] found, in patients with asthma and chronic obstructive pulmonary disease (COPD), that the incidence of falls was more prevalent in the COPD group, with $32 \%$, compared with $28 \%$ in patients with asthma and patients with polytherapy, depression symptoms, hospitalizations, coronary disease, dementia, and diagnosis of COPD or asthma have a higher risk of falling.

\section{DIAGNOSIS}

For the diagnosis, clinical history and physical examination are essential, combined with an electrocardiogram, chest X-ray, and spirometry [15]. The diagnosis of asthma requires assessment of lung function with spirometry, and demonstration of variability by bronchodilator or corticosteroid reversibility, airway hyperresponsiveness [16]. The reversibility of airway obstruction in elderly asthmatic patients is often reduced because of irreversible structural changes resulting from airway remodeling [17].

The FEV1/FVC ratio decreases with aging, and therefore, it is essential to use age-adjusted values. With aging, bronchial hyperresponsiveness to methacholine increases; therefore, provocation testing may be less accurate in this age group [18]. Bronchoprovocation challenges may be contraindicated in some older patients with low baseline lung function or cardiac comorbidities. Spirometry involves effort-dependent maneuvers and it may be difficult for those who are frail. Poor coordination and muscle weakness in some patients may produce inaccurate readings of peak expiratory flow [19].

Other tests such as the carbon monoxide diffusing capacity of the lung may help distinguish between asthma and COPD. Chest computed tomography may demonstrate increased wall thickness, and increased air-trapping in older patients with asthma [20]. The use of exhaled nitric oxide in older patients is not completely established, and aging itself can increase exhaled nitric oxide [21]. Some studies using exhaled nitric oxide as a marker for asthma diagnosis and a measure of control in older patients have shown it to be beneficial, whereas others have not [22].

Measurement of plasma brain natriuretic polypeptide and assessment of cardiac function with echocardiography may also be helpful. In patients with a history of smoking or biomass fuel exposure, COPD and overlapping asthma and COPD should be considered [15].

\section{TREATMENT}

The treatment of asthma is enforced at the control of the symptoms and reducing exacerbations [23].

Table 3. Main factors associated with asthma

\begin{tabular}{llll}
\hline Pharmacologic & Comorbidities & Physiologic & Pulmonary changes \\
\hline Drugs interactions & Obesity & Depression & Fixed airway \\
Increased risk of side-effects & Diabetes mellitus 2 & Anxiety & Increase in small airway disease \\
Inappropriate medication usage & Hypertension & Cognitive impairment & Increased susceptibility to infections \\
& Coronary artery ischemia & Impaired sensation of & FEV1/FVC ratio decreases \\
& Gastritis, ulcer, gastroesophageal & dyspnea & \\
& reflux & & \\
& Tobacco use & & \\
& Cognitive impairment & \\
\hline
\end{tabular}

FEV1, forced expiratory volume in 1s; FVC, forced vital capacity.

Modified with permission from $\left[5^{\mathbf{m}}\right]$. 
Asthma is characterized by chronic airway inflammation disease. Therefore, inhaled corticosteroids are the treatment of choice [16].

The inhaled route is the best option for efficacyto-safety treatment of asthma; in the elderly, the technique is a problem that may lead to poor disease control, associated with cognitive impairment and reduced lung function in patients [5"']. Older patients may have difficulties with complex medication regimens, and prescription of multiple inhaler devices should be avoided if possible [15].

The efficacy of asthma medications in the elderly is limited. Side-effects of $\beta 2$-agonists such as cardiotoxicity, and corticosteroid side-effects are more common in the elderly [15]. In the management of patients with LOA is of most importance taking comorbidities into account because a high number of prescribed medications may increase the risk of drug interactions, negative impact on efficacy, safety, and worsen adherence with controller medication for asthma.

Psychologic diseases like depression and anxiety in patients with LOA are important; they have been associated with poor outcome in the management, including higher exacerbation rate and mortality [16].

Westerhof et al. [17] determined the remission rate and identified predictors of persistent adultonset asthma and found that patients with persistent asthma were older, had worse asthma control, required higher doses of inhaled corticosteroids, had more severe airway hyperresponsiveness, more often presented nasal polyps, and higher levels of blood neutrophils as compared to patients who experienced clinical remission.

\section{DISCUSSION}

Unlike younger adults and children with asthma who encounter overdiagnosis and in some cases an excess in treatment, older people with asthma continue to present the highest mortality and are underdiagnosed which may imply not receiving the most adequate therapy measures [15]. Previous reviews have considered as well the challenges of treating asthma in older people because of the confusion of distinguishing between comorbidities such as COPD or gastroesophageal reflux disease which may converge with asthma in a later lifetime period [24]. Along with the comorbidities are risk factors and distinct clinical outcomes, described previously by Bennet et al. [4] in which independent risk factors associated with fixed air obstruction include male sex, older age, and increased duration of asthma [25].

\section{CONCLUSION}

Asthma in the elderly is a disease that negatively affects the functionality and QOL of individuals. The lack of diagnosis associated with the changes of aging in the respiratory system and the multiple comorbidities make it difficult to establish treatments effective for its control. Current evidence shows that the elderly benefit from a stepped treatment, as do young patients with asthma, requiring the monitoring of the adverse effects of both corticosteroids and bronchodilators to ensure good control and therapeutic adherence. In view of the demographic and epidemiological transition, it is necessary that physicians from all branches dedicated to health of the elder form multidisciplinary teams that ensure quality medical care.

\section{Acknowledgements}

None.

\section{Financial support and sponsorship}

This work was supported by the Regional Center of Allergy and Clinical Immunology. Universidad Autonoma de Nuevo León, Facultad de Medicina, University Hospital 'Dr José Eleuterio González'. Monterrey, Nuevo León, Mexico.

No funding was received for this work from National Institutes of Health (NIH); Wellcome Trust; Howard Hughs Medical Institute (HHMI); or others.

\section{Conflicts of interest}

There are no conflicts of interest.

\section{REFERENCES AND RECOMMENDED}

\section{READING}

Papers of particular interest, published within the annual period of review, have been highlighted as:

- of special interest

-1. of outstanding interest

1. Cavalazzi R, Jorayeva $A$, Beatty $B$, et al. Predicting asthma in older adults on - the basis of clinical history. Respir Med 2018; 142:36-40.

This article provides a diagnostic accuracy study using methacholine challenge test to obtain positive predictors for asthma in elderly patients.

2. Papi A, Brightling C, Pedersen SE. Asthma. Lancet 2018; 391:783-800.

3. Pasha MA, Sundquist B, Townley R. Asthma pathogenesis, diagnosis, and -1. management in the elderly. Allergy Asthma Proc 2017; 38:184-191.

Pasha et al. highlight the challenges presented by physicians regarding the burden of high medical costs and also emphasize the high mortality in elderly patients with asthma.

4. Bennet $\mathrm{GH}$, Carpenter L, Hao W, et al. Risk factors and clinical outcomes associated with fixed airflow obstruction in older adults with asthma. Ann Allergy Asthma Immunol 2018; 120:164-168.

5. Dunn RM, Busse PJ, Wechsler ME. Asthma in the elderly and late-onset adult -1. asthma. Allergy 2018; 73:284-294.

This article by Dunn et al. provides accurate, recent, and useful data regarding agerelated physiologic and immunologic changes in elderly patients with asthma, providing a better understanding of the underlying causes and mechanisms involved.

6. Polivka BJ, Folz R, Myers J, et al. Identifying phenotypes and factors impacting outcomes in older adults with asthma: a research protocol and recruitment results. Research in Nursing and Health 2018; 41:336-345.

7. Nagaratnam N, Nagaratnam K, Cheuk G. Asthma in the elderly. Geriatric diseases. Evaluation and Management. 1st edition. Springer International Publishing; 2010; 99-104. 
8. Baptist AP, Busse PJ. Asthma over the age of 65 : all's well that ends well. J Allergy Clin Immunol: In Practice 2018; 3:764-773.

9. Pawalec G. Age and immunity: what is immunosenescence? Exp Gerontol 2018; 105:4-9.

10. Fulop $T$, Larbi A, Dupuis $G$, et al. Immunosenescence and inflamm-aging as two sides of the same coin: friends of foes? Front Immunol 2018; 8:1960.

11. Braman SS. Asthma in the elderly. Clin Geriatr Med 2017; 33:523-537.

12. Gemicioglu $B$, Bayram $H$, Cimrin A. Asthma control and adherence in newly diagnosed young and elderly adult patients with asthma in Turkey. J Asthma 2018; 6:1-9.

13. Yáñez $A$, Soria $M$, De Barayazarra $S$, et al. Clinical characteristics and comorbidities of elderly asthmatics who attend allergy clinics. Asthma Res Pract 2018; 4:5.

14. Bozek A, Jarzab J, Hadas $E$, et al. Fall episodes in elderly patients with asthma and COPD: a pilot study. J Asthma 2018; 8:1-5.

15. Ulrik CS. Late-onset asthma: a diagnostic and management challenge. Drugs Aging 2017; 34:157-162.

16. Hirano $T$, Matsunaga K. Late onset asthma: current perspectives. J Asthma Allergy 2018; 11:19-27.

17. Westerhof GA, Coumou H, de Nijs SB, et al. Clinical predictors of remission and persistence of adult-onset asthma. J Allergy Clin Immunol 2018; 141:104-109.
18. Vaz Fragoso CA, McAvay G, Van Ness PH, et al. Phenotype of normal spirometry in an aging population. Am J Respir Crit Care Med 2015; 192:817-825

19. Luoto JA, Elmstahl $S$, Wollmer $P$, et al. Incidence of airflow limitation in subjects 65-100 years of age. Eur Respir J 2016; 47:461-472.

20. Inoue $H$, Niimi A, Takeda $T$, et al. Pathophysiological characteristics of asthma in the elderly: a comprehensive study. Ann Allergy Asthma Immunol 2014; 113:527-533.

21. Toren $\mathrm{K}$, Murgia N, Schioler L, et al. Reference values of fractional excretion of exhaled nitric oxide among nonsmokers and current smokers. BMC Pulm Med 2017; 17:118.

22. Malerba M, Damiani G, Carpagnano GE, et al. Values in elderly people for exhaled nitric oxide study. Rejuv Res 2016; 19:233-238.

23. Global Initiative for Asthma. Global Strategy for Asthma Management and Prevention 2018 (cited 2018 Oct 31). Available from: https://ginasthma.org/ 2018-gina-report-global-strategy-for-asthma-management-and-prevention/. [Accessed 1 October 2018]

24. Gibson PG, McDonald VM, Marks GB. Asthma in older adults. Lancet 2010; 376:803-813.

25. Zhang L, He L, Gong J, et al. Risk Factors Associated with Irreversible Airway Obstruction in Asthma: A Systematic Review and Meta-Analysis BioMed Research International 2016; 2016. 